dass insbesondere Fatigue, Infusionsreaktionen, Exantheme und Autoimmunreaktionen (Hepatitis, Pneumonitis, Kolitis) vorkommen und somit einen Klasseneffekt der Substanzgruppe darstellen.

Fatigue wird als eine der häufigsten relevanten Nebenwirkungen von onkologischen Patienten mit signifikanten Einschränkungen der Lebensqualität berichtet - dagegen deutlich seltener von den Ärzten als relevante Toxizität eingeschätzt. Onkologen sollten daher die Fatigue ernst nehmen, die den Patienten im Alltag, im Haushalt, im Berufsleben und in der Familie erheblich belastet. Die Therapiemöglichkeiten (Bewegung, Ernährung und Life-style-Modifikation, Medikamente) sind jedoch begrenzt. Eine besondere Bedeutung hat aber auch die sogenannte finanzielle Toxizität. Dies wurde insbesondere auf der ASCO-Jahrestagung 2015 in Chicago thematisiert. In Anbetracht der Jahrestherapiekosten von 100.000 Euro und mehr ist die Frage nach Kosten und Nutzen hochrelevant. Insbesondere wenn eine Übernahme durch Kostenträger nicht oder nur zum Teil gewährleistet ist. Allerdings gehört diese Diskussion nicht in das Arztzimmer, wo die medizinisch op- timale Therapie mit dem Patienten besprochen werden soll.

Die Immunonkologie steht noch am Beginn der klinischen Entwicklung - mit mehr Hoffnungen als fertigen Ergebnissen. Viele Fragen sind noch offen: Welches sind die relevanten tumorspezifischen Antigene? Gibt es Prädiktoren für das Therapieansprechen, wie z. B. den histologischen Nachweis von PD1/PD-L1 im Gewebe? Was ist die optimale Methode in der Immunonkologie: Checkpointblockade? Bi- oder multispezifische Antikörper? Vakzinationen gegen tumorspezifische Antigene? Oder zelluläre Immuntherapiestrategien? Ist eine Kombinations- der Monotherapie überlegen? Wie können Therapieresistenzen überwunden oder gar verhindert werden?

Angesichts von weltweit ca. 8,2 Millionen Menschen, die an Krebs versterben, kommt die sogenannte "Moonshot"-Initiative von US-Präsident Obama und Vizepräsident Biden zur rechten Zeit. Noch nie waren die Bedingungen im Kampf gegen den Krebs günstiger als heute: Die modernen molekulargenetischen Analysen ermöglichen eine rasche Bestimmung von tumorrelevanten genetischen Mutationen im Serum und da- durch in bestimmten Fällen eine personalisierte Therapie mit zielgerichteten Substanzen. Sie ersparen vielleicht in Zukunft invasive Biopsien im Falle eines Rezidivs oder bei Verdacht auf Metastasen. Bei Subgruppen von Patienten mit bestimmten Tumoren hat die Immuntherapie ein großes Potenzial für Langzeitremissionen gezeigt. Die nächsten Jahre werden die Immuntherapie als die vierte Säule der Krebsbehandlung etablieren und die Therapie mit großer Wahrscheinlichkeit revolutionieren.

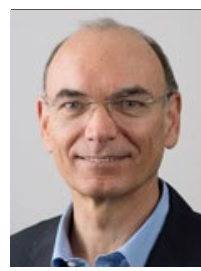

Prof. Dr. med Hans Tesch Centrum für Hämatologie und Onkologie Bethanien, Frankfurt hans.tesch@telemed.de

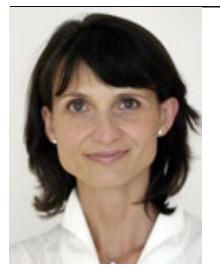

Dr. med. Christina Moisidis-Tesch Gynäkologische Gemeinschaftspraxis, Wiesbaden c.moisidis-tesch@ gynaekologiewiesbaden.de

\title{
Wie Komorbiditäten die Therapieentscheidung beeinflussen
}

\section{Viele Krebspatienten leiden unter Komorbiditäten. Die Therapie- entscheidung sollte hier in multi- disziplinären Teams erfolgen.}

- ntscheidungen über die Krebstherapie Ewerden zunehmend in multidisziplinären Tumorboards gefällt. In einem systematischen Review wurde nun untersucht, wie Komorbiditäten in solchen Meetings berücksichtigt werden und die Therapieentscheidungen beeinflussen. Ausgewertet wurden 21 Studien, in denen zwischen 2006 und 2014 TeamMeetings in Großbritannien, Australien, Hongkong, Frankreich, Schweden und Deutschland untersucht wurden. Dabei zeigte sich, dass Komorbiditäten in vielen Meetings nicht ausreichend berücksichtigt wurden. Sie wurden oftmals nicht routinemäßig betrachtet und diskutiert, es sei denn, ein Teammitglied identifizierte sie als Einflussfaktor für die
Therapieentscheidung. Das Vorliegen bzw. Fehlen von Informationen über Komorbiditäten beeinflusst die Therapieentscheidung: Komorbiditäten erschwerten die Erstellung eines klaren Therapieplans, sodass häufig keine eindeutigen Therapieentscheidungen getroffen wurden. Wurden bei Patienten mit Komorbiditäten Therapieempfehlungen gegeben, entsprachen sie häufig nicht den Leitlinien. Patienten mit mindestens einer Begleiterkrankung bekamen häufiger keine Therapie. Zudem wurden die im Team getroffenen Therapieempfehlungen bei Patienten mit Komorbiditäten häufig nicht umgesetzt: Die tatsächliche Behandlung war oft konservativer als die empfohlene Therapie, auch wenn es Evidenz für deren Verträglichkeit und Wirksamkeit gab. Mitglieder multidisziplinärer Team-Meetings sind sich wahrscheinlich nicht bewusst, dass Komorbiditäten bei ihren Entscheidungen igno- riert werden. Die Forscher empfehlen, Komorbiditäten in multidisziplinären Tumorboards systematisch zu betrachten. Allerdings darf dies nicht zu Unteroder Übertherapie führen, auch wenn die Balance schwierig zu halten ist. In weiteren Untersuchungen sollte festgestellt werden, wie Komorbiditäten bei Therapieempfehlungen angemessen berücksichtigt werden können. Dies könnte die klinischen Ergebnisse von Krebspatienten mit Komorbiditäten verbessern.

Fazit: Das Vorliegen bzw. Fehlen von Informationen über die Komorbiditäten hat wesentlichen Einfluss auf die Therapieentscheidung. Künftig sollten Komorbiditäten in Tumorboards stärker berücksichtigt werden. Judith Neumaier

Stairmand J et al. Consideration of comorbidity in treatment decision making in multidisciplinary cancer team meetings: a systematic review. Ann Oncol. 2015;26(7):1325-32. 